\title{
Germanica
}

„Wir spüren nämlich nichts mehr, werte Dame“ (Selbst-)Entfremdung bei Melle, Genazino und Von Steinaecker

"Wir spüren nämlich nichts mehr, werte Dame“ - (Self-)Alienation in Melle, Genazino and Von Steinaecker

„Wir spüren nämlich nichts mehr, werte Dame“ - Aliénation et dépossession de soi chez Melle, Genazino et Von Steinaecker

\section{Cora Rok}

\section{OpenEdition}

\section{Journals}

Édition électronique

URL : http://journals.openedition.org/germanica/2669

DOI : 10.4000/germanica.2669

ISSN : 2107-0784

\section{Éditeur}

Université de Lille

\section{Édition imprimée}

Date de publication : 30 décembre 2014

Pagination : 111-126

ISBN : 9782913857346

ISSN : 0984-2632

\section{Référence électronique}

Cora Rok, « „Wir spüren nämlich nichts mehr, werte Dame“ - (Selbst-)Entfremdung bei Melle, Genazino und Von Steinaecker », Germanica [Online], 55 | 2014, Online erschienen am: 30 Dezember 2016, abgerufen am 06 Oktober 2020. URL : http://journals.openedition.org/germanica/2669 ; DOI : https:// doi.org/10.4000/germanica.2669 


\section{„Wir spüren nämlich nichts mehr, werte Dame“ - (Selbst-)Entfremdung bei Melle, Genazino und Von Steinaecker}

Cora RoK

Rheinische Friedrich-Wilhelms-Universität Bonn

Titulierte Ingeborg Bachmann eines ihrer Debütgedichte, „Entfremdung“1 (1948), noch selbstverständlich mit einer Bezeichnung für ein Phänomen, das von Jean-Jacques Rousseau im 18. Jahrhundert sozialphilosophisch im Zusammenhang mit einer Anthropologie und Staatslehre theoretisiert, bevor es von Georg Friedrich Hegel in seiner Phänomenologie als notwendige Stufe zur Geistesausbildung verstanden und schließlich Ende des 19. Jahrhunderts in Karl Marxens Arbeitsphilosophie zu einem antikapitalistischen Kampfbegriff gegen ausbeuterische Verhältnisse bei Industriearbeitern erweitert wurde, zweifelt Protagonistin Laura in Thomas Melles 2011 erschienenen Roman Sickster an diesem ideologisch aufgeladenen und ambivalenten Begriff: „,Entfremdung', dachte sie. Das gibt es doch gar nicht. Das darf es doch nicht geben, so ein Wort. Eine marxistische Erfindung ist das, ein hegelianisches Konstrukt, semantischer

1. - Ingeborg Bachmann, „Entfremdung“, in: ders., Sämtliche Gedichte, München, Piper, 2003 (1948). 
Müll der Frühmoderne, seit Generationen weitergereicht" 2 . Bildete die Entfremdung in der Existenzphilosophie des 20. Jahrhunderts (Kierkegaard, Heidegger, Sartre) noch eine wesentliche Kategorie zur Beschreibung moderner Seinserfahrungen - und hier schließt sich Bachmann, die 1949 über Heidegger promovierte, noch an - verebbte das Interesse an dem brisanten Schlagwort, nachdem postmoderne und poststrukturalistische Theorien die vorherrschende Subjektvorstellung dekonstruiert und die Einheitlichkeit des Individuums und dessen autonome Handlungsverantwortlichkeit in Frage gestellt hatten. Melle, im Übrigen auch Philosophieabsolvent, lässt Laura mit dieser Aussage also zurecht an einen aktuellen Diskurs anschließen und gar erschüttert den Bedeutungshorizont des Entfremdungsbegriffs hinterfragen. Dass Entfremdung dennoch Hauptthema des Romans und auch wiederkehrendes Motiv in anderen zeitgenössischen Werken nicht nur der Belletristik, aber auch der Soziologie ${ }^{3}$, Philosophie ${ }^{4}$ und Psychologie ${ }^{5}$ ist, lässt vermuten, dass der ,,semantische Müll“" durchaus ein Phänomen ist, dem nach wie vor Wirkkraft zugesprochen werden kann. Dabei gilt es, die Frage nach der „Neuheit“ von entfremdenden „Prozessen“ innerhalb der Gesellschaft (soziale Ursprünge) sowie „Zuständen“ der Entfremdung (psychische Konstitutionen) zu stellen. Während gegenwärtig die Philosophin Rahel Jaeggi und der Soziologe Hartmut Rosa den Entfremdungsdiskurs anti-essentialistisch rehabilitieren, kann die Literaturwissenschaft literarische Dokumente auf Formen der Entfremdung hin untersuchen und die Mittel ihrer Darstellung prüfen, um nicht nur eine Linie literarischer Tradition ziehen (oder unterbrechen) zu können, sondern gegebenenfalls auch aus den Repräsentationen der Entfremdungsszenarien und Bewusstseinszuständen Rückschlüsse auf gesellschaftliche Prozesse ziehen zu können, die zumindest zunächst innerhalb der fiktiven Welt für entfremdungsfördernde Entwicklungen verantwortlich gemacht werden können. Dieser Beitrag möchte sich, um das Forschungsfeld zu verengen, exemplarischen Romanen der deutschen Gegenwartsliteratur, im Besonderen Melles Roman widmen, bei denen der thematische Komplex um die zeitgenössische Arbeitswelt ein verbindendes Element darstellt und in dessen Rahmen Entfremdung eine Rolle spielt.

2. - Thomas Melle, Sickster, Berlin, Rowohlt, 2011, S. 65f. 2013.

3. - Vgl. Hartmut Rosa, Beschleunigung und Entfremdung, Berlin, Suhrkamp,

4. - Vgl. Rahel Jaeggi, Entfremdung. Zur Aktualität eines sozialphilosophischen Problems, Frankfurt am Main, Campus, 2005.

5. - Vgl. Matthias Michael, Depersonalisation und Derealisation: Die Entfremdung überwinden, Stuttgart, Kohlhammer, 2012. 


\section{„Ich weisz, dasz ich wahr werden kann. Ich musz nur genügend wollen"6. - Thomas Melles Sickster (2011)}

In Melles Roman ist der Titel Programm: Sickster, eine Mischung aus sick (krank) und Hipster, eine Bezeichnung des amerikanischen Schriftstellers Norman Mailer in seinem 1957 veröffentlichten Aufsatz „The White Negro“ für den „American existentialist“, der die Leitideen der Selbstbestimmung und Individualität der bornierten „Spießergeneration“ der 50er Jahre entgegenstellt ${ }^{7}$, verweist auf das Wesen eines neuen Subjekttypus, der sich vornehmlich in der Medienund Kreativwirtschaft betätigt, dessen originäre Ideale sich aber zu seinem Leid gegen ihn gekehrt haben. Die Verpflichtung zur Autonomie und die Verantwortung im Kampf um Authentizität, die auch JeanPaul Sartre als existentialistisches Konzept entwickelte ${ }^{8}$, scheinen postmoderne Arbeitssubjekte in pathologische Krankheitsbilder zu führen. Dem Typus Sickster entsprechen nun Thomas Melles Protagonisten, der Industriejournalist Magnus Taue und der Manager Thorsten Kühnemund, die beide für denselben Ölkonzern arbeiten und vornehmlich Tankstellenpächtern Trendberichte und Instruktionen zur ,umsatzsteigernde[n] Neustrukturierung und Optimierung von Shopbereichen"9 zukommen lassen. Die dritte Figur bildet die bereits zitierte Laura, betrogene und emotional vernachlässigte Freundin des erfolgreichen Sex-maniac ${ }^{10}$ Thorsten, die eine Stelle als Assistentin ihres Juraprofessors erwartet, sich aber im Call-Center verdinglicht und unbefriedigende Fortbildungsseminare besucht.

Die Psychogramme der Figuren zeichnend, changiert Melle Erzählebenen, um mal aus auktorialer Erzählinstanz zu charakterisieren und mal durch Tagebucheinträge (Laura) mit RechtschreibVerfremdungen - stets wird das Doppel-S zu einem „,sz“, und Diktiergerätaufnahmen (Magnus) Bewusstseinsströme in ihrer Unmittelbarkeit sprechen zu lassen. Aber auch in die vermeintlich objektive Erzählinstanz mischt sich eine subjektive Note, sodass Erzähler- und Figurenstimme miteinander verschmelzen und eine Form der erlebten

\footnotetext{
6. - Melle, S. 209.

7. - Vgl. Norman Mailer, „The White Negro“, http://www.dissentmagazine.org/ online_articles/the-white-negro-fall-1957 (zuletzt aufgerufen am 16.9.2014).

8. - Vgl. Jean-Paul Sartre, L'être et le néant. Essai d'ontologie phénoménologique, Paris, Gallimard, 1976 (1943).

9. - Melle, S. 36.

10. - Ebd., S. 34 .
} 
Rede $^{11}$ bilden ${ }^{12}$. Resultat ist eine erzählerische Inkohärenz, die sich durch das rasche Wechseln von Erzählperspektiven, durch Brüche im semantischen Register und dem Schwanken zwischen präzisen, poetischen und lakonisch, vulgären oder umgangssprachlichen Wendungen oder auch Einfädelungen von Liedzitaten bildet und an den Versuch eines therapeutischen Schreibens zur Bestimmung der Ursache scheiternder Schicksäle erinnert.

Beständig werden die psychischen Dispositionen der Charaktere entweder von ihnen selbst oder vom kommentierenden Erzähler gedeutet. Thorsten wird als „negativer Möglichkeitsmensch“ und „Augenblicksjongleur“, stets on the run, ,im Modus eines ständig sich erneuernden Präsens, das die Zukunft nur als Fluchtpunkt"13 kennt, beschrieben und erfüllt somit die Anforderung an den "flexiblen Menschen" der Arbeitswelt des 21. Jahrhunderts, dem Richard Sennett trotz gesteigerter Selbstständigkeit und Kontrollgewinn im beruflichen Feld, gerade durch die Abnahme langfristiger Verbindlichkeiten Kontroll- und Sinnverlust im Privaten attestiert ${ }^{14}$. Dass Thorsten die Möglichkeit einer anderen Seinsweise jenseits des abwechslungsgierigen und aufzehrenden Lebensstils nur im Ansatz bedenkt oder gar keine Kriterien zu einer Bewertung aufzustellen vermag, wird deutlich, beachtet man die Häufigkeit der Passagen, in denen die Charaktere ihre Selbstreflexivität unter Beweis stellen; während in den Laura- und Magnus-Kapiteln die Figuren als Ich-Erzähler versuchen, ihr Leiden zu diagnostizieren, nimmt bei Thorsten der Erzähler die Auslegung vor und erläutert, was „mit Thorstens Bewusstsein passiert“ war ${ }^{15}$. Ökonomischer Wettbewerbsgeist und Leistungsdrang korrelieren mit einer Verdinglichung und Funktionalisierung auch im Bereich des Intimen, Skrupel und Zweifel aufgrund der Abwesenheit gültiger Werte scheint es nicht zu geben. Auch als der zunehmende Alkoholismus Thorstens Leistungs- und Selbstdarstellungsfähigkeit nicht mehr stimuliert, sondern ihn seinen Job kostet, kann er sich Handlungsschwäche nicht eingestehen: ,,...] wenn er wollte, wäre er nüchtern und gesund;

11. - Stanzel spricht von einer ,, Ansteckung' der Erzählersprache durch die Figurensprache“, vgl. Franz Stanzel, Theorie des Erzählens, Göttingen, Vandenhoeck und Ruprecht, 1995, S. 25.

12. - Vgl. Melle, S. 35.

13. - Ebd., S. 32.

14. - Vgl. Richard Sennett, The Corrosion of Character: The Personal Consequences of Work in the New Capitalism. London / New York, Norton \& Company, 1999.

15. - Vgl. Melle, S. 34: „Er war durchsexualisiert worden [...]. Er wusste nicht, wie normal oder gut oder schlecht es war, es war einfach. Er sah eine Frau, sie wurde zum mentalen Pin-up [...].“ 
dessen war er sich gewiss“16. Das „Wollen“ und die „wahre Existenz“ ist auch bei Laura Thema: „Ich weisz, dasz ich wahr werden kann. Ich musz nur genügend wollen" 17 , schreibt sie in ihr Tagebuch, doch scheint ihr die Formulierung eines erstrebenswerten Zieles und eine dahin ausgerichtete Selbstverwirklichung nicht möglich, sodass sie gar nicht dazu kommt, ihr Handlungspotential zu erkennen. Stattdessen liefern ihre Aufzeichnungen stets Hinweise darauf, wie sie auf Thorstens Annäherungen (oder Abweisungen) nur passiv „,reagiert“". Dabei zieht sie den Rückzug in die Innerlichkeit (,einfach liegen und schlafen und weg sein, jetzt, wo er da war" ${ }^{18}$ ) der aktiven Gestaltung vor. Auch Magnus fürchtet die Entschlossenheit: „Er wollte, dass ihm alles Gute zustoßen möge, alle Wünsche wahr würden, ohne dass ein Wille in ihm keimte und er somit Schuld auf sich laden könnte"19. Die Bequemlichkeit der Ziellosigkeit und moralischen Rückgratlosigkeit und andererseits die ökonomische Notwehr, lassen ihn aus seiner gegenwärtigen Rolle, mit der er sich nicht identifizieren kann, nicht austreten:

Natürlich dachte er auch irgendwie an die Zukunft, natürlich hatte auch er irgendetwas Richtiges vor, zum Beispiel, endlich seinen Film in Angriff zu nehmen, den er so lange schon plante, aber er mochte es auch, durch die Tage zu driften, ohne Ziel, ohne die sogenannten Perspektiven, und die Jobs anzunehmen, wie sie eben halbwegs kamen ${ }^{20}$.

„Eigentlich“aber, glaubt Magnus seine wahre, künstlerische Berufung als größter Filmemacher in spe $e^{21}$ durch die berufliche Tätigkeit, die nicht ,,seinen Moralmaßstäben von sich selbst“22 entspreche, verfehlt zu haben. Sein Leben unter der Gewissheit ,talentiert“ zu sein und den „Ruf eines Genies“ genossen zu haben, was in ihm ein Überlegenheitsgefühl trotz Schwäche reifen lässt, in der Defensive verbringend, schafft er es nicht, konstruktiv zum Akteur von Handlungen zu werden, die seinem Ideal-Ich entsprechen. Auch Laura leidet unter der Diskrepanz zwischen Ideal- und Ist-Zustand: „Das Wort, das ich am meisten hasse, ist das Wort ,eigentlich““23. Dabei verweist die Rede von der „Eigentlichkeit“, die nach Heidegger ein durch verantwortungsvolle Entscheidungen

16. - Ebd., S. 310 .

17. - Ebd., S. 209.

18. - Ebd., S. 65. Gerade dieses stumme Erdulden löst bei Thorsten auch einen „Hass“ auf Laura aus, der die Unaufrichtigkeit noch weiter provoziert. Vgl. ebd., S. 118.

19. - Ebd., S. 145.

20. - Ebd., S. 175.

21. - Ebd., S. 247.

22. - Ebd., S. 229.

23. - Ebd., S. 113. 
geprägtes Dasein jenseits gesellschaftlicher Konformität bezeichnet ${ }^{24}$, auf die Suche nach Authentizität, die dem gesamten Roman zugrunde liegt.

Alle drei Figuren sind auf der Suche nach dem großen Gefühl - sich selbst erleben sie als ,,jemand anderes“ ${ }^{25}$, als ,,falsch“ und ,gekünstelt“ 26 oder „,fremd“ 27 , häufig fallen Worte wie „,imitieren“, „,modulieren“ und „mimen“, zwischenmenschliche Nähe und affektives Betroffensein werden nur ,simuliert“28. „Wir spüren nämlich nichts mehr, werte Dame“, lässt Thorsten, der stets seine Arbeitsperformance als „Rockstar des Absatzes und der Verkaufskonzepte“ 29 im Fokus hat, sich gerne mit Energydrinks und vom „Jargon“ der Businesswelt „,berauscht“30 und sich in Alkoholeskapaden und Affären abreagiert, die Barfrau wissen, als er sich und seinem Kollegen eine hochprozentuale Mixtur beordert: „Wir sind stumpf. Wir brauchen's mit dem Vorschlaghammer"31.

Eine kalte Distanz herrscht zwischen den vermeintlich Liebenden Thorsten und Laura, statt dem erwarteten Gefühl stellt sich Müdigkeit ein:

Er konnte das alles noch immer sehen, ihre Schönheit und deren Details [...] - aber er konnte ihre Schönheit nicht mehr fühlen. Etwas von dieser Schönheit ging verloren auf dem Weg zu ihm, etwas, das Entscheidende. / Ihr Anblick machte ihn müde. Das war neu. / Es drang nicht zu ihm durch, genau wie die Fakten dieses Ortes, der Schutt des Weltkriegs, die Ruinen des Kalten Krieges, er konnte sich das aufsagen, aber es sagte ihm nichts. Empfindungslose Erinnerung ${ }^{32}$.

Auch der Ort, an dem die beiden picknicken, der Berliner Teufelsberg, ruft nur Teilnahmslosigkeit hervor, obgleich hier der Rohbau der nationalsozialistischen Wehrtechnischen Fakultät überschüttet und später als Abhörstation der Amerikaner genutzt wurde. Langeweile, Gefühlsleere und Gleichgültigkeit paaren sich bei Laura gar mit Dissoziationen und Angstzuständen, die in einer Abspaltung des Geistes vom Körper münden.

Während sich Personen und Dingwelt und ihr affektiver und sinnstiftender Bezug Laura und Thorsten entziehen und beide pathologische Rückholbewegungen zur Spürbarmachung des Selbst entwik-

24. - Vgl. Martin Heidegger, Sein und Zeit, Berlin, De Gruyter, 2006 (1927).

25. - Melle., S. 48.

26. - Ebd., S. 98f.

27. - Ebd., S. 67; S. 95.

28. - Ebd. S. 72.

29. - Ebd., S. 86.

30. - Ebd., S. 71.

31. - Ebd., S. 45.

32. - Ebd., S. 83. 
keln - Thorsten in der Erregung beruflicher Herausforderungen (was im Burn-Out kulminiert) sowie im Drogen- und Alkoholmissbrauch und Laura ebenfalls in Betäubungsmitteln, der masochistischen Aufrechterhaltung einer „Beziehungs-Simulation“33, die sie zermürbt, und in der Selbstverletzung, mit der sie schon als Kind Aufmerksamkeit $\mathrm{zu}$ erheischen hoffte ${ }^{34}$ - bedrängen diese Magnus auf bedrohliche Art und Weise: „Zu viele Sinneseindrücke prasselten auf ihn ein, vor allem, wenn er von anderen Menschen umgeben war. [...] Er bedachte zu viel, blieb in der Außenperspektive stecken, fühlte sich, den anderen vermeintlich bis in die letzte Faser seiner Gedankentextur durchschauend, gelähmt“35. Nicht nur „die Aura industrieller Perfektion und gelbstichiger Supermarktstrenge“"36, auch die

Tags und Graffiti, sonst unbeachtet am Rande des Blickfeldes, drängten sich auf, wollten ausgesprochen werden. Seine Stimme klang jedoch umso fremder, je lauter er redete. [...] Ausgeweidete Telefonzellen standen im Weg. Gesprächsfetzen verhöhnten ihn. [...] Alles ging ihn an. Alles griff ihn an ${ }^{37}$.

Lebensekel $^{38}$ überfällt die Protagonisten und das semantische Feld des „Klebrigen“, das auch Jean-Paul Sartre in La Nausée wählt, in dem Roquentin die „Gegenwart als Konfitüre“39 bezeichnet, wird an mehreren Stellen ${ }^{40}$ aufgegriffen. „Alle diese Gegenstände....sie belästigen mich; ich hätte gewünscht, sie würden weniger stark existieren,

33. - Ebd., S. 213.

34. - „Die Lüge aber war schon so übermächtig vorhanden in Laura, in ihrem Körper, dass sie diese kleine Stelle Wahrheit offenhalten wollte, so lange es ging, diese Wunde“. Ebd., S. 198. Auch entwickelt Laura eine weitere Methode, um zu der „kalten, feindlichen Mondlandschaft“ ihres Zimmers einen Bezug herzustellen: „Um irgendetwas Persönliches, Besitzergreifendes zu tun, hatte sie ihre Monatsblutung nicht aufgefangen und so, in einer betrunkenen Nacht, Laken und Matratze großflächig markiert." Ebd., S. 70.

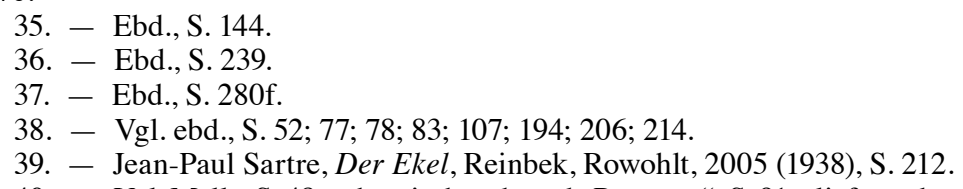

40. - Vgl. Melle, S. 48: „,chemisch wabernde Prozesse“; S. 91: „liefen zäh wie alter Honig“; S. 179: ,alles buk zusammen zu einem klumpigen Brei, wie zähe, erkaltende Lava. Erstarrte.“; S. 187: „Namen [...] vermengen sich zu einem unauflöslichen Brei“; S. 93: „klebriger Gedankenfaden“; S. 127: „Prozentkuchen waberten vor Thorstens Gesichtsfeld“; S. 128: ,ihre Form ein Wabern... und der Monitor absorbierte das Licht... sein Magen drehte sich um...und es roch nach vergorenem Honig...“; S. 144: „Kacheln wabern“; S. 145: „wabbelig wie Austernfleisch“; S. 205: „von derselben neutralen, klebrig freundlichen Offenheit“; S. 280: ,waberte ein ZACK!“; S. 296: ,gegen klebrige Widerstände“. 
auf trockenere, abstrakte Weise, mit mehr Zurückhaltung ${ }^{641}$, schreibt Roquentin in sein Tagebuch und fühlt sich selbst von den ,dicken rauhen Pranke[n] der Wurzeln“ gestört, die für ihn „Beispiel nackter Existenz, Symbol des Daseins ohne Vergangenheit und Zukunft" $\operatorname{sind}^{42}$. Auch Magnus kann der Natur ,nichts abgewinnen“, sodass ihn gar das „Gewisper der Bäume in einem Park“"43 belästigt. Aurel Kolnai definiert in seinem 1929 erschienenen philosophischen Aufsatz Der Ekel eben diesen als passive Abwehrreaktion eines Subjekts auf dessen direkte Affizierung und unterscheidet zwischen einem physischen Ekel (vor Fäulnis oder Verwesung, aber auch vor dem ,wuchernden Leben“ wie im Beispiel der Bäume) und moralischen Ekel (Falschheit, Korruption, moralische Schwäche), wobei die Konturlosigkeit, die sich in den literarischen Beispielen im Bild des Klebrigen, Wabernden und Breiigen ausdrückt, das Verbindende zwischen den beiden Formen darstellt ${ }^{44}$. Gerade unter der Konturlosigkeit des Daseins und der eigenen Passivität und Gestaltungsohnmacht leidet Magnus. Doch nicht nur Sartres Roquentin, sondern auch die Protagonisten der Romane Alberto Moravias teilen sein Schicksal; handlungsgehemmt, aber hochintellektuell, gefühlsarm, aber idealistisch, suchen sie aufzubegehren gegen die bürgerliche Lebenswelt, deren Konformismus, schale Zerstreuungssucht und Doppelmoral. Doch nie bringen sie die nötige Motivation und Rechtfertigung zustande, die sie zu einem Ausbruch aus der noia (Langeweile), indifferenza (Gleichgültigkeit), nausea (Ekel) und angoscia (Angst) ${ }^{45}$ befähigen würde. Mino, der gutbürgerliche Student aus La Romana, würde gerne Ziele haben, für die es sich zu kämpfen lohnt, stellt aber fest, dass, als er sich mit der Unterschicht identifizieren will, sein politisches Engagement für ihn selbst (über den Egozentrismus wächst er nicht hinaus) keine relevante Veränderung bringen würde und daher nicht authentisch ist ${ }^{46}$. Sein Selbstmord bleibt die einzig rebellische Geste.

Ähnlich desorientiert bei der Übersetzung geistiger Energien in Handlungen, aber vor allem unter wachsender Isolation und Paranoia leidend, liefert sich Magnus schließlich selbst in eine Anstalt (in der

41. - Sartre, S. 201. Gleichzeitig leidet Roquentins Geliebte Anny unter einem „Zuwenig“ an Gefühl und setzt sich zum ersten Kuss in die Brennnesseln, um überhaupt Erregung zu spüren, vgl. ebd., S. 235.

42. - Ebd., S. 147.

43. - Melle,. S. 144.

44. - Aurel Kolnai, „Der Ekel“, in: Edmund Husserl (Hrsg.): Jahrbuch für Philosophie und phänomenologische Forschung, Halle, 1974 (1929), S. 515-569, hier S. 525.

45. - Vgl. Joachim Leeker, Existentialistische Motive im Werk Alberto Moravias.

Ein Vergleich mit Malraux, Camus und Sartre, Bensberg, Schäuble, 1979.

46. - Vgl. Alberto Moravia, La Romana, Marchio, Bompiani, 2005 (1947). 
auch Laura landet) ein, wo ihm nahegelegt wird, er ,hätte so etwas wie eine Kreuzung oder Mischung dieser beiden Krankheitsbilder gehabt, Zyklothymie und Schizophrenie, und das war dann das. Stoffwechsel. Neuronen. Botenstoffe“ ${ }^{47}$. Der vitale Impuls zur Wiederaneignung der in die Ferne gerückten Lebenswelt kommt bei Magnus nun in der institutionellen Ausgrenzung, die ihm eine gewisse närrische Freiheit des Denkens jenseits ökonomischer Zielsetzungen ermöglicht, sodass sein „Wunsch nach Veränderung dieses Systems“ sowie der „Zwang“, die eigenen Hände von der „ölverschmierten Schuld reinzuwaschen“48, sich in einer „Flash-Mob“-Revolte der aufgestachelten Klinikpatienten gegen den Ölkonzern entlädt ${ }^{49}$. Obgleich angesichts des willensstarken und moralisch motivierten Engagements von Magnus auch Laura aus dem „Dornröschenschlaf“ “50 erwacht, sich gar zwischen den beiden Zärtlichkeiten ergeben („Laura hatte dabei das erste Mal seit langem eine Empfindung, [...]. Sie fühlte etwas. / Sich, unter anderem. / Das war neu. “51), stürzt sich Magnus nach dem gelungenen Hoax vom Dach des Konzerns in die Tiefe.

Ist nun die in Bachmanns Gedicht geschilderte Distanz des lyrischen Ichs zur Welt, die ihre Sinnhaftigkeit verloren zu haben scheint, seine Hoffnungslosigkeit und die Möglichkeit des ,sich wieder Näherns“ mit der Form von Entfremdung zu vergleichen ist, die Melles Figuren zum Großteil in die Psychiatrische Klinik treibt? Während das Gedicht, drei Jahre nach Kriegsende verfasst, durchaus die Sinnkrise und Hoffnungslosigkeit, den Werteverfall und die (geistige) Heimatlosigkeit angesichts der Gräuel des Zweiten Weltkriegs einfängt und Entfremdung in diesem Kontext einen konkreten Auslöser zu haben scheint, kann aber auch die mangelnde Responsivitätserfahrung mit der natürlichen Umgebung als existenzielle Angst vor einer „stummen, repulsiven Welt“, vor dem „Verlust der Antwort- oder Resonanzbeziehung“ und dem „Indifferent-Werden“52 verstanden werden, die als epochenübergreifende Konstanten anzuerkennen sind. Auch die literarischen Bilder

47. - Melle, S. 223.

48. - Ebd., S. 309.

49. - Dieses Motiv des Aufstandes gegen einen Großkonzern findet sich auch in Markus Henriks Copy Man, Frankfurt am Main, Eichborn, 2009, wo die ausgebeuteten Praktikanten, denen die Übernahme verwehrt wurde, ebenfalls in einem Flash-Mob das Unternehmensgebäude stürmen. Verbindend ist auch die ,Impotenz' des Charakters Anton in Copy Man und Magnus, die beide in der Situation des bevorstehenden Geschlechtsakts von überrationalen Gedanken gehemmt werden.

50. - Melle, S. 305.

51. - Ebd., S 317.

52. - Hartmut Rosa, „Kritik der Zeitverhältnisse. Beschleunigung und Entfremdung als Schlüsselbegriffe einer erneuerten Sozialkritik“, in: Rahel Jaeggi (Hg. u.a.), Was ist Kritik?, Frankfurt am Main, 2009, S. 23-54, S. 11. 
der Fremdheitserfahrung bei Bachmann, Sartre, Moravia und Melle stellen eine geistige Nähe her. Gleich bleibt ebenfalls die Aussicht auf eine mögliche Versöhnung, die „Wieder-Annäherung“ an die eigene Lebenswelt, das aktive Sich-in-Beziehung-Setzen zu den Dingen. Werden jedoch Nachkriegsgesellschaft oder Bourgeoisie zum Auslöser für Lebensekel, Depersonalisations- sowie Derealisationserfahrungen, deuten Melles Protagonisten ihren Weltschmerz und Handlungsunfähigkeit letztlich nicht konsequent als soziale Pathologie der Leistungs- und Sensationsgesellschaft, sondern als Krankheit des „Stoffwechsels“53 und Störung des Nervensystems und lassen sich klinisch behandeln, womit sie die Schuld der „Unangepasstheit“ an die Gesellschaft bei sich selbst suchen. Während Sartre und Moravia noch politisches oder schriftstellerisches Engagement als möglichen Ausweg aus der Entfremdung feierten, der politische Herd in Mitteleuropa während ihrer Autorentätigkeit noch brodelte, so wirkt das in Sickster beschriebene Aufbegehren gegen den mächtigen Ölkonzern wie ein Tropfen auf dem heißen Stein - ,ändern würde sich nichts, nie“54. Dass das Vorhaben der intrinsisch motivierten Selbstverwirklichung zum Scheitern verurteilt ist, zeigt nicht nur Titel und Ausgang von Melles Roman, sondern auch der Blick auf weitere zeitgenössische Romane, deren Handlung im neoliberalen Arbeitsdiskurs angelegt ist und aus denen eine Poetik des Pathologischen extrahiert werden kann.

\section{„Auf diesem eigentlich beruht das halbe Leben!“655 - Wilhelm Genazinos Das Glück in glïcksfernen Zeiten (2009)}

Obwohl Gerhard Warlich, dessen sprechender Name auf seinen Konflikt anspielt, über Heidegger (!) promoviert hat und sich „eigentlich“ als „Philosoph“, „Ästhet“, „stiller Kommunikator“ oder gar „Konzeptkünstler" 56 sieht, arbeitet er seit 14 Jahren in einer Großwäscherei, wo er nach dem Studium zwecks schneller Rückzahlung des Studienkredits zunächst als Ausfahrer angefangen hatte und bald zum Chef aufgestiegen war. Warlich lebt in einer

53. - Während Laura ihre Kondition auf biologische Ursachen zurückführt (vgl. Melle, S. 208), versucht Magnus bspw. ,psychopathologische Störungen“ auf einen Tinnitus zurückzuführen, der für die Instabilität des Nervensystems verantwortlich gemacht werden könnte, vgl. ebd., S. 13.

54. - Ebd., S. 329.

55. - Ebd., S. 19.

56. - Wilhelm Genazino, Das Glück in glücksfernen Zeiten, München, Hanser, 2009, S. 19. In seinem Habitus erkennt Warlich stets einen Verweis auf sein „eigentliches“ Künstlerdasein, selbst das zerfranste Unterhemd, als „Symbol für die Marterungen des Lebens“, wird zu einem „Hinweis auf [s]eine Zukunft als Künstler. Ich würde gerne (wenn es so etwas gibt) ein Kleiderkünstler werden, besser: ein Verwesungskünstler.“ Ebd., S. 18. 
Großstadt, sehnt sich ,eigentlich“ nach einem anderen Lebensort, er hat eine Lebenspartnerin, mit deren Bedürfnissen (nach einem Kind) und ,starken Gestaltungsdrang, dessen Opfer auch [er] zuweilen“57 wird, er „eigentlich“ gar nicht zurechtkommt, sodass er resignierend feststellt: „Auf diesem eigentlich beruht das halbe Leben!“58 Warlich gibt zu, lediglich ein „Beinahe-Künstler“ zu sein, der zwar Collagen macht, zeichnet, malt, filmt und schreibt, ,,aber nichts davon so richtig“ ausübt, nicht ,leidenschaftlich und also ohne Ausweg, jedenfalls nicht so, daß ich mich (wie jetzt wieder) alle drei bis vier Wochen fragen muß, was wirklich in mir steckt" ${ }^{\prime 59}$. Warlich erlebt eine „metaphysische Bestürzung "60 angesichts wachsender Unfreiheiten, die nicht nur von seiner Arbeitsstelle ausgehen - die seinem intellektuellen Anspruch nicht genügt und von der er sich als „Spitzel“ missbrauchen lässt, indem er den Ausfahrern hinterherspioniert, um zu prüfen, ob sie nicht privaten Angelegenheiten während der Arbeitszeit nachgehen -, sondern von seinem, ihm als feindlich gegenübertretenden Umfeld gemeinhin, das sich als einflussreicher erweist als seine Sehnsüchte, ,etwas ganz und gar Richtiges tun zu wollen" 61 und einen Beruf zu finden, der ihm „endlich die Gewißheit verschafft“, sich ,,in einem sinnvollen Leben“ $z u$ befinden ${ }^{62}$. Schon auf den ersten drei Seiten lässt das vom Ich-Erzähler verwendete Vokabular ${ }^{63}$ auf seinen Zustand schließen: die Wirklichkeit, so wie er sie wahrnimmt, und sein Leben sind für ihn eine ,unhaltbare Sache“64; ,Ich höre jetzt nur noch das Wehklagen meiner ratlosen Seele. Sie möchte gern etwas erleben, was ihrer Zartheit entspricht", erklärt Warlich und sieht einen Straßentrompeter, der mit seinem erbettelten Geld eine Flasche Cognac kauft, was seine „Seele mächtig, aber ergebnislos" aufbäumen lässt ${ }^{65}$. Trost findet Warlich zunächst im Anblick von Ameisen und im weiteren Verlauf generell in der Kontemplation des

57. - Ebd. S. 16. „Ich komme nur nicht damit zurecht, daß ich mich fortlaufend zu ihr verhalten muß.“ Ebd., S. 19.

58. - Ebd., S. 19.

59. - Ebd., S. 17.

60. - Ebd., S. 41.

61. - Ebd., S. 42.

62. - Ebd., S. 14.

63. - „Nur mit Mühe“, die „Sonne scheint schwach“, „ausgepumpte, reglose“ Menschen, „Leistungsgesellschaft“, „Müdigkeit“, „Mitleid“ und „Bedauern“ der Bedienungen, „Scham“ über die „Konsum-Parolen auf meinen beiden Plastiktüten“, „Überempfindlichkeit“, „öffentliche Peinlichkeit“, „Mißtrauen“, „Erschöpfung“, „,vertrautestes Unbehagen“, „Wunsch nach Veränderung“, „,stumm bleibenden Drang, die Menschen über die allgemeine Ödnis des Wirklichen aufklären zu wollen“, „kläglich alles Geschehende“, „öffentliche Armseligkeit“, „voll innere Ablehnung“, „innere Stummheit" etc., vgl. ebd. S.7ff.

64. - Ebd., S. 8.

65. - Ebd., S. 9f. 
tierischen Daseins („Die Unbesorgtheit der Tiere rührt mich“66) sowie in der Beobachtung einer Verrückten, in deren Wahnsinn er ,etwas Belebendes und Wunderbares“ erkennt und die ,in die Halbtoten“, die sie betrachtenden Cafébesucher, ,hineinstößt“ und ihre „Bedürftigkeit“ und ,Erschöpfung“"vertreib ${ }^{\text {t67 }}$. Gerade aber diese Erscheinung zu Beginn der Erzählung antizipiert Warlichs Zukunft. Warlichs liebste, aber realitätsferne Eigenart nämlich, das Beobachten und „Phantasieren“, ist ihm einziges „Glück“; die „halb-außerirdischen Vorgänge“, denen er „im Laufe [s]eines Beobachterlebens“ gewahr werden konnte, sprich, die Erkenntnis und Verknüpfung der „Koinzidenz der Ereignisse“, vermögen es, Warlich zu „faszinieren, trösten“ und zu „beruhigen“68. Ein ,universale[s] Lebenseinverständnis“69, „Heiterkeit“70 oder ein „erwärmt[es] Herz"71 erfährt Warlich dann, wenn er über die im Radio gefallenen Namen eines Baritons (Heinrich Schlusnus) und der Sprecherin (Astrid Redlich) „,herumempfindeln“ kann, „,ohne daß dabei irgend etwas herauskommen müßte"; als er den Einfall entwickelt, eine trockene Scheibe Brot in seiner Brieftasche aufzubewahren, um diese anstelle eines Geldscheines jemandem entgegenzuhalten; und als er über das utopische „Projekt >Halbtags leben<“ phantasiert. Die an kreativen und humorvollen Einfällen reiche „Innenwelt“, die sich „,mehr und mehr vor die Außenwelt schiebt"72, kollidiert allerdings zunehmend mit Warlichs sozialer Lebenswirklichkeit, die seiner sichtbaren Entfremdung mit wachsender Beunruhigung entgegenblickt. Nachdem Warlich während seiner Arbeitszeit als (zufälliger) Zuschauer einer Anarchisten-Demo gesichtet und anschließend gefeuert wird, führt ihn seine psychische Instabilität in die Psychiatrie. Auch hier kann er nicht Herr über seine Entscheidungen werden und lässt die Versuche der Kontaktaufnahme durch andere Patienten duldsam über sich ergehen, bis er von der beglückenden Erkenntnis durchfahren wird, dass auch er „trotz allem, immer noch wählen [kann]“, wie er „in Zukunft leben will“"73. Dass am Ende die Aussicht auf die gegenwärtige Optionenvielfalt steht, aber keine Handlung im Sinne einer Entscheidung „für etwas“ erfolgen kann, verweist auf dieselbe Problematik der Verwirklichung der „Eigentlichkeit“, die auch in Thomas von Steinaeckers Roman thematisiert wird.

66. - Ebd., S. 135.

67. - Ebd., S. 11.

68. - Ebd., S. $12 \mathrm{f}$.

69. - Ebd., S. 17.

70. - Ebd., S. 43.

71. - Ebd., S. 59.

72. - Ebd., S. 121.

73. - Ebd., S. 158. 


\section{„Ich brauchte den Wettkampf als Motivation“674 - - Thomas von Steinaeckers Das Jahr, in dem ich aufhörte mir Sorgen zu machen und anfing zu träumen (2012)}

Mit der sozialen Rolle, in die Warlich und Magnus durch die (mehrjährige) Tätigkeit in einem Unternehmen, die sie patent aber unleidenschaftlich ausüben, hineingewachsen sind, können sie sich nicht identifizieren, was zu einer Selbst- und Weltentfremdung und schließlich zu extremen Pathologien führt 75 . Dagegen scheint Thomas von Steinaeckers Protagonistin Renate Meißner durch die Kündigung noch knapp einem Burn-Out und einer Selbstaushöhlung zu entgehen. Im Unterschied zu Warlich und Magnus über-identifiziert sich Renate Meißner mit ihrer angeeigneten Rolle als stellvertretende Abteilungsleiterin in einem Münchner Versicherungskonzern und stellt zu Anfang des Romans deren Adäquatheit nicht in Frage (womit sie Thorsten ähnelt). Renate Meißner - ebenso studierte Philosophin - braucht den ,Wettkampf als Motivation"76. Sie ermahnt sich mit groben Schimpfworten zur Leistungssteigerung („Manchmal half mir das beim Fokussieren“"77) und erstellt sich allabendlich „Performance-Eigenevaluationen“78, die es ihr erlauben, sich ,hinsichtlich Orientierung bzgl. der Abläufe“, „hinsichtlich der eigentlichen Tätigkeit, d.h. Leichenbeseitigung, Neupolicierung, Akquise etc.“, „,hinsichtlich der eigenen Darstellung, d.h. von Renate Meißner, d.h. mir selbst, d.h. Kommunikation meiner Position gegenüber Kollegen bei Wahrung eines emotional angemessenen Verhältnisses“, und ,hinsichtlich Abwehr von in diesem Rahmen Nebensächlichem, aber Nichtsteuerbarem, d.h. z.B. Erinnerungen“79 selbst zu kontrollieren. Das „Nichtsteuerbare“ allerdings, das Moment des Zufälligen, Unkontrollierbaren, ist dabei der größte Störfaktor. Dazu gehören vor allem Gefühle, die durch Erinnerungen heraufbeschwört werden; Erinnerungen an die erniedrigende Beziehung zu ihrem damaligen Chef, dem Familienvater Walter, und offene Fragen zu dem mysteriösen Verschwinden der Großmutter. Renate Meißner entwikkelt deshalb ein emotionales Selbstregulierungsprogramm und erstellt „Kausaldiagramme“, „ein in dem Seminar »Lebenslogik« gelerntes, probates Instrument, um über komplexe Verläufe in der eigenen

74. - Thomas von Steinaecker: Das Jahr in dem ich aufhörte mir Sorgen zu machen und anfing zu träumen. Frankfurt am Main, Fischer, 2012, S. 29.

75. - Vgl. Jaeggi: Entfremdung, S. 63-182 (Kap. II ,,Sein eigenes als ein fremdes Leben leben: Vier Fälle“).

76. - Von Steinaecker, S. 29.

77. - Ebd., S.19.

78. - Ebd., S. 42.

79. - Ebd., S. 42. 
Biographie, die auf denersten Blick wirr scheinen, Klarheitzu erlangen" 80 . Wirtschaftsvokabular wirkt sich auf Renates Wahrnehmungshorizont aus, sie taxiert die Menschen in ihrer Umgebung, also hauptsächlich ihre Kollegen, um versicherungstechnische Prognosen zu erstellen, zieht „Bilanzen“81 über den vergangenen Party-Abend und verlässt sich auf Statistiken, um sich in Bezug auf ihr Leben Berechen- und Planbarkeit zu suggerieren. Wenn sich bei Frau Meißner trotz Einnahme eines beruhigenden Antidepressivums ein „Flattern im Brustbereich“82 einstellt, und sie sich „100\% souverän lächelnd“ von der „schwitzigfeucht gewordenen Oberfläche“ der Empfangstheke der Firma löst, die ihr zuvor „Halt gegeben“ hatte ${ }^{83}$, verweist dies allerdings auf die Kluft zwischen gewünschtem und zur Schau gestellten professionellen Selbstbild und tatsächlicher Befindlichkeit. In dem Moment, in dem sie ihre Selbstbeherrschung verliert - sie rutscht zu Hause auf dem Parkett aus - treten all jene unterdrückten Emotionen an die Oberfläche, die sich in einem „Schütteln des Körpers vor Schluchzen“, das ihr ,immer besonders fremd" vorkommt, artikulieren ${ }^{84}$. Professionelle Contenance ist für Renate ein Schutzschild, der sie davor bewahrt, Schwäche zu zeigen und sie sich überhaupt einzugestehen.

Leben bedeutet für Steinaeckers Protagonistin die Verpflichtung zur permanenten Selbstüberholung und Optimierung, ihre Ansprüche treiben sie dabei an ihre psychischen Grenzen. Laut Hartmut Rosa lauere gerade im Autonomieversprechen die Gefahr des Burn-Outs und des Sinn- und Selbstverlusts. Trotz selbstauferlegter Aufgaben erfahren sich die eigenverantwortlichen Subjekte als „Dauerversager“ und fürchten, Aufgaben nicht bewältigen zu können und dadurch abgehängt zu werden, wodurch Gefühle der „Fernsteuerung“ und Ohnmacht entstehen ${ }^{85}$. In den Momenten, in denen ihre rationale Selbst- und Lebenskonstruktion ins Wanken gerät, wird sich Frau Meißner ihrer Selbstentfremdung und ihrem Mangel an nichtverdinglichenden Interaktionsverhältnissen mit anderen Menschen gewahr. Die Selbstsuche, die Erkundung der ,eigentlichen' Bedürfnisse und die Verarbeitung der Familiengeschichte rund um das mysteriöse Verschwinden der Großmutter lassen Renate von Möglichkeiten eines anderen, besseren Lebens (nicht auf die ,Kalorien zu achten, ohne ,Ziel' spazieren zu gehen, ohne ,Grund' auf der Wiese zu sitzen, ,Liebe', ,Glück ${ }^{6}$ und ,Sinn` zu erfahren ${ }^{86}$ ) ,träumen'. Am

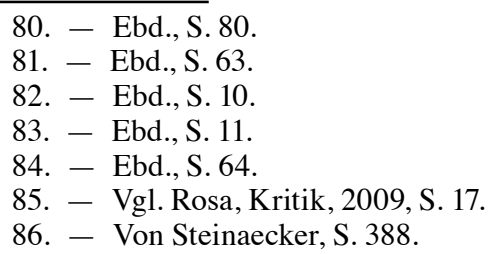


Ende des Romans versucht Renate, aus der Rolle einer ehrgeizigen und selbstbeherrschten Selbstunternehmerin durch die Kündigung herauszutreten, doch mit der neugewonnenen Freizeit konfrontiert, beginnt sie, die Verwirklichung ihrer Träume durch strategische Pläne wiederum in berechenbare Strukturen zu pressen ${ }^{87}$.

\section{Postmoderne Entfremdung oder moderne Entfremdung in postmodernen Arbeits- und Lebensverhältnissen?}

Nicht nur Melles, Genazinos und Von Steinaeckers Roman bilden interessante Forschungsobjekte für die Entschlüsselung gegenwärtiger literarischer Darstellungen von Entfremdungsformen. Blickt man auf weitere Texte, die sich in den letzten Jahren vermehrt dem Thema Arbeit im 21. Jahrhundert widmen und von (autobiographisch inspirierten) Praktikanten- ${ }^{88}$, Call-Center- 89 und Büroromanen ${ }^{90}$, literarisierten Arbeitnehmerinterviews ${ }^{91}$, hin $\mathrm{zu}$ Narrativen der Arbeitslosigkeit ${ }^{92}$, der Kreativwirtschaft ${ }^{93}$, der Kosmetik-94 oder weiteren Unternehmerbranchen ${ }^{95}$ reichen, so spielt auch in diesen die Entfremdungsthematik eine ausschlaggebende Rolle. Dabei lassen sich in den Romanen zwei Typen von Protagonisten unterscheiden; diejenigen, die ihre Rolle in der Arbeitsgesellschaft erfüllen und bei denen Formen der Entfremdung nur für den Leser sichtbar sind (Thorsten, Renate), und diejenigen, die mit hoher Selbstreflexivität bereits ihre Rolle selbst als entfremdend erleben (Laura, Magnus, Warlich). Während bei letztgenannten der Entfremdungsdiskurs noch nah an der Moderne liegt und an marxistische Kritik an der mangelnden Aneignungsmöglichkeit und Selbstständigkeit sowie dem Gefühl der

87. - Während Renate letzten Endes ebenso wie Warlich mit der Qual der Wahl der Lebensgestaltung konfrontiert ist, sieht Magnus die einzige Freiheitshandlung in seinem Freitod.

88. - Vgl. Markus Henrik, Copy Man, Frankfurt am Main, Eichborn, 2009; Boris Fust, Zwölf Stunden sind kein Tag, München, Piper, 2008.

89. - Vgl. Sebastian Thiel, Call Center. Wer dranbleibt, hat verloren, Berlin, Schwarzkopf \& Schwarzkopf, 2012.

90. - Vgl. Anne Weber, Gold im Mund, Frankfurt am Main, Suhrkamp, 2005.

91. - Vgl. Kathrin Röggla, wir schlafen nicht, Frankfurt am Main, Fischer, 2006.

92. - Vgl. Jakob Hein, Herr Jensen steigt aus, München, Piper, 2006; Annette Pehnt, Mobbing, Piper, München, 2007; Joachim Zelter, Schule der Arbeitslosen, Tübingen, Klöpfer und Meyer, 2006. 2001.

93. - Vgl. Rainer Merkel, Das Jahr der Wunder, Frankfurt am Main, Fischer,

94. - Vgl. Philipp Schönthaler, Das Schiff das singend zieht auf seiner Bahn, Berlin, Matthes \& Seitz, 2013.

95. - Vgl. Ernst-Wilhelm Händler, Wenn wir sterben, Frankfurt am Main, Frankfurter Verlagsanstalt, 2002; Rainald Goetz, Johann Holtrop, Berlin, Suhrkamp, 2012. 
Fragmentiertheit, der Fremdbestimmung und Fremdausbeutung erinnert, so lässt die Subjektvorstellung des neuen unternehmerischen (kreativ-)Arbeiters das Problem der Entfremdung in einem anderen Licht erscheinen; die Kritik an der Entfremdung verschiebt sich zur Kritik an der Selbstausbeutung, dem Annehmen fremder Motivationen als eigene sinnstiftende Zielsetzungen durch die Aufwertung der Autonomie und der totalen Vereinnahmung des Arbeitnehmers. Dabei führt die Verschmelzung von privater und Arbeitssphäre und somit die Ökonomisierung aller Lebensbereiche nicht nur zu einem Mangel familiärer Geborgenheit, zu Kommunikationsunfähigkeit, Vereinzelung sowie Verdinglichung personeller Beziehungen, sondern vor allem zu Burnout und Depression, neuen Formen von Entfremdung, die durch eine (Über-)Identifikation mit der Arbeitsrolle entstehen (Thorsten, Renate). Die „Künstlerthematik“, die sich in den Literaturbeispielen durch die Figuren Magnus und Warlich widerspiegelt, die Sehnsucht nach einem kreativen Selbstausdruck, der nicht ökonomischen Imperativen folgt und der hohen intellektuellen Qualifizierung angemessen ist, die Suche nach Authentizität und Wahrhaftigkeit führt zu einem weiteren Themenkomplex, der „Krankheit des Wollens“, die die Protagonisten daran hindert, ideelle Vorstellungen in aktive Gestaltung zu übersetzen, sodass sich Formen des Selbstbetrugs oder „mauvaise foi“ (Sartre) bilden, an denen die existentialontologische Theorie ansetzen kann. Dass innerhalb der Fiktion aller postmodernen Diskurse zum Trotz von „Eigentlichkeit“ und ,authentischem“ Ich, dessen Entfaltung durch gesellschaftliche (oder chemisch-biologische Ungleichgewichte!) verhindert wurde, gesprochen wird, muss nicht von einer Fixierung auf essentialistischen Subjektvorstellungen zeugen, obgleich die Nähe des Duktus der literarischen Beispiele zu ihren Vorgängern es nahelegt; Rahel Jaeggi rettet den Entfremdungsbegriff gerade in unser heutiges Subjektverständnis, indem er auf ,,,pathologische“ Formen des Rollenverhaltens“" verweist, die den „Entwicklungsprozess“ von einem „falschen" zu einem, allerdings stets fluide bleibenden, ,wahren Selbst“, das sich „,nur in der gelingenden Identifikation mit Rollen bilden kann“, verhindern ${ }^{96}$. Bezeichnend ist für die Gegenwartsliteratur, dass diese Thematik in zeitgenössische Arbeitsverhältnisse eingebettet wird, die einen immensen Einfluss auf die Lebensbiographien der Subjekte haben. 